\title{
Browning of white adipose tissue after a burn injury promotes hepatic steatosis and dysfunction
}

\author{
Abdikarim Abdullahi ${ }^{1,2}$, Osai Samadi ${ }^{1,2}$, Christopher Auger ${ }^{2}$, Tharsan Kanagalingam ${ }^{2}$, Darren Boehning ${ }^{3}$, Sheng Bi ${ }^{4}$ and \\ Marc G. Jeschke (iD ${ }^{1,2,5,6}$
}

\begin{abstract}
Burn patients experiencing hypermetabolism develop hepatic steatosis, which is associated with liver failure and poor outcomes after the injury. These same patients also undergo white adipose tissue (WAT) browning, which has been implicated in mediating post-burn cachexia and sustained hypermetabolism. Despite the clinical presentation of hepatic steatosis and WAT browning in burns, whether or not these two pathological responses are linked remains poorly understood. Here, we show that the burn-induced WAT browning and its associated increased lipolysis leads to the accelerated development of hepatic steatosis in mice. Deletion of interleukin 6 (IL-6) and the uncoupling protein 1 (UCP1), regulators of burn-induced WAT browning completely protected mice from hepatic steatosis after the injury. Treatment of post-burn mice with propranolol or IL-6 receptor blocker attenuated burn-induced WAT browning and its associated hepatic steatosis pathology. Lipidomic profiling in the plasma of post-burn mice and burn patients revealed elevated levels of damage-inducing lipids (palmitic and stearic acids), which induced hepatic endoplasmic reticulum (ER) stress and compromised hepatic fat oxidation. Mechanistically, we show that hepatic ER stress after a burn injury leads to a greater ER-mitochondria interaction, hepatocyte apoptosis, oxidative stress, and impaired fat oxidation. Collectively, our findings uncover an adverse "cross-talk" between the adipose and liver tissue in the context of burn injury, which is critically mediated by WAT browning.
\end{abstract}

\section{Introduction}

Hypermetabolic reprogramming is considered a hallmark of burns and has been an area of accelerated research over the last decade. A common theme emerging from this work is that when nutrients are scarce, metabolic signaling pathways direct enhanced nutrient acquisition via the mobilization of lipids and proteins ${ }^{1,2}$. This mobilization may result in cachexia, which is associated with the loss of muscle proteins, and alterations in fat metabolism including a substantial induction of lipolysis. While muscle catabolism and hepatic steatosis are well documented in the pathophysiology of burn injury, the involvement of the white adipose tissue (WAT) in this process is essentially unknown. Recently, however, two

\footnotetext{
Correspondence: Marc G. Jeschke (marc.jeschke@sunnybrook.ca) ${ }^{1}$ Faculty of Medicine, University of Toronto, Toronto, ON, Canada ${ }^{2}$ Biological Sciences, Sunnybrook Research Institute, Toronto, ON, Canada Full list of author information is available at the end of the article. Edited by I. Amelio
}

independent studies related to the metabolic consequences of cancer and burns described the phenomenon of WAT browning in human and rodent models, in which subcutaneous white adipocytes convert to a phenotypically similar brown-like adipocyte, termed beige/ brite adipose tissue ${ }^{3-5}$.

During burn-induced WAT browning, systemic elevations in catecholamines lead to the activation of the $\beta_{3^{-}}$ adrenergic receptor, which stimulates lipolysis and subsequently increases the expression of UCP1 within white adipocytes $^{4,6,7}$. Of great importance is the link between WAT lipolysis and browning. Lipolysis is almost ubiquitously present in burn patients and has been associated with increased insulin resistance, and most importantly, fatty acid infiltration in essential organs, such as the liver and kidney ${ }^{8-10}$. Furthermore, in both cancer patients and patients suffering from cardiovascular diseases, browning of WAT and its associated increased lipolytic state has been implicated in facilitating organ steatosis (muscle and

\section{(c) The Author(s) 2019}

(c) (i) Open Access This article is licensed under a Creative Commons Attribution 4.0 International License, which permits use, sharing, adaptation, distribution and reproduction cc) in any medium or format, as long as you give appropriate credit to the original author(s) and the source, provide a link to the Creative Commons license, and indicate if changes were made. The images or other third party material in this article are included in the article's Creative Commons license, unless indicated otherwise in a credit line to the material. If material is not included in the article's Creative Commons license and your intended use is not permitted by statutory regulation or exceeds the permitted use, you will need to obtain permission directly from the copyright holder. To view a copy of this license, visit http://creativecommons.org/licenses/by/4.0/. 
a
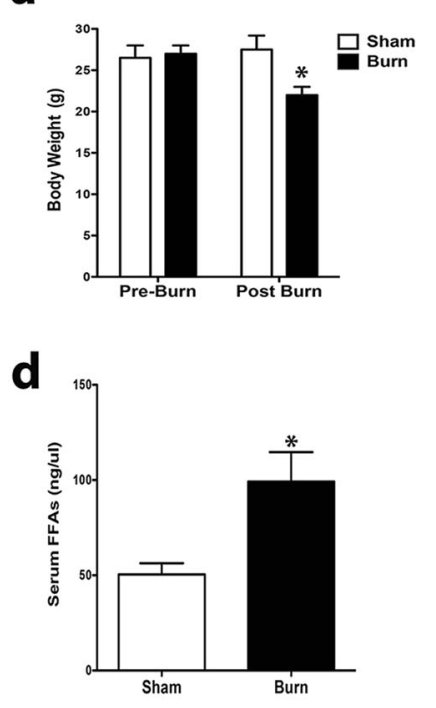

g

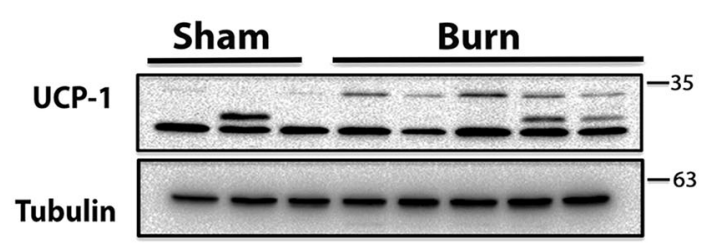

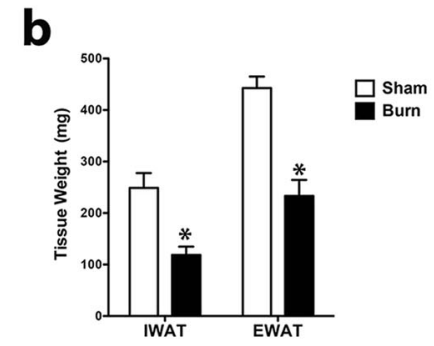
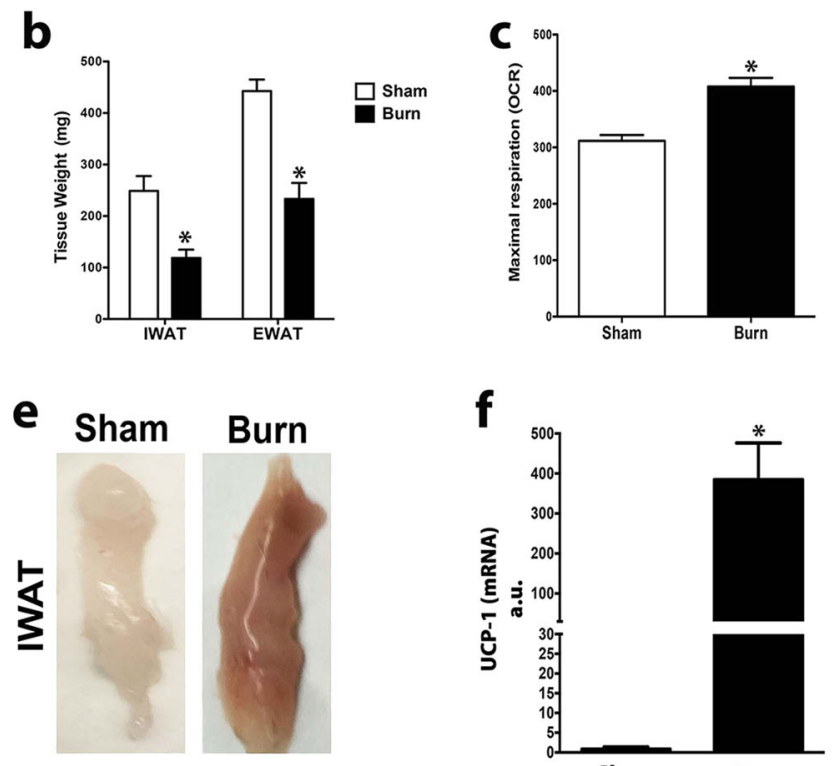

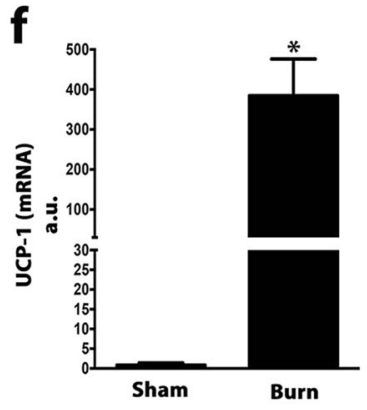

h Sham Burn

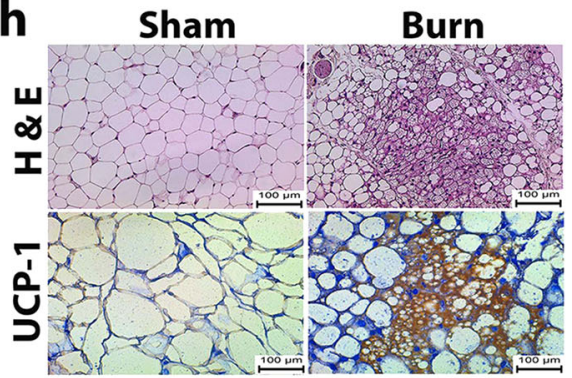

Burn
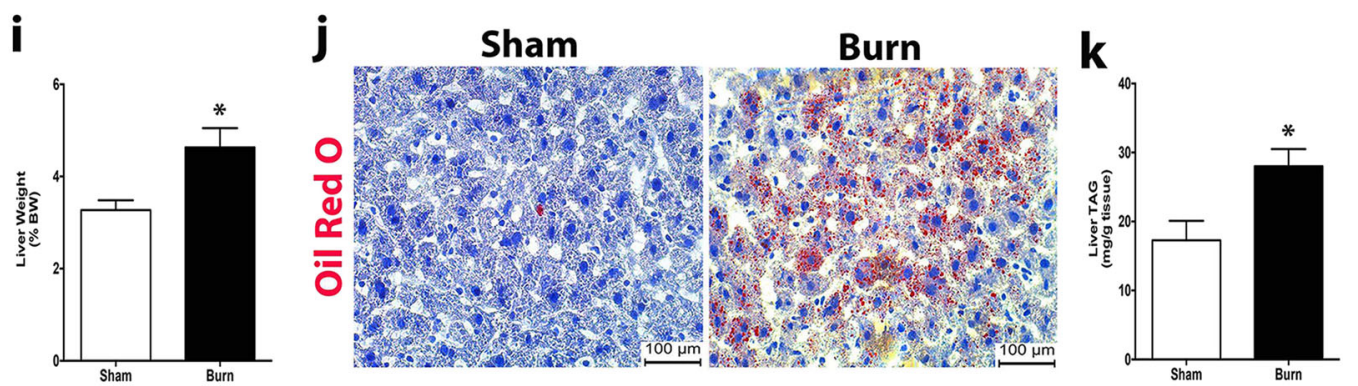
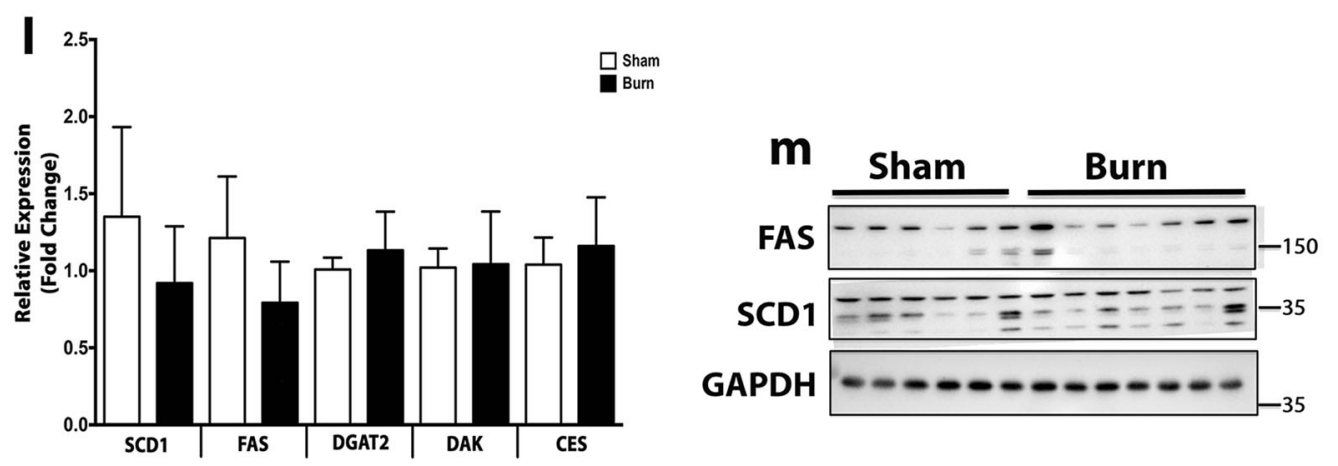

Fig. 1 (See legend on next page.) 
(see figure on previous page)

Fig. 1 Browning of white adipose tissue leads to the development of hepatic steatosis post-burn injury. a, b Changes in total body (a) and adipose tissue (b) weight in post-burn and control mice. c Analysis of oxygen consumption rate in isolated inguinal WAT of post burned mice and controls. $\mathbf{d}$ Plasma concentration of free fatty acids in burned mice and controls. e Representative macroscopic pictures of isolated inguinal WAT from burned mice and controls at autopsy. $\mathbf{f}$ Quantitative RT-PCR analysis of browning gene Uncoupling protein 1 (UCP1) in inguinal WAT of burned mice and controls. $\mathbf{g}$ Immunoblot analysis of UCP1 in inguinal WAT of burned mice and controls. $\mathbf{h}$ H\&E and UCP1 staining in inguinal WAT of burned mice and controls. i Liver weights normalized to body weight of burned mice and controls. $\mathbf{j}$ Oil Red O staining for fat droplets in liver sections from burned mice and controls. $\mathbf{k}$ Triglyceride (TG) content of livers from burned mice and controls. I Quantitative RT-PCR analysis of lipogenic genes was measured in livers from burned mice and controls. $\mathbf{m}$ Immunoblot analysis of lipogenic proteins in livers from burned mice and controls. Data represented as mean \pm SEM, $p<0.05$ *significant difference burn vs. controls ( $n=8$, biological replicates, experiments repeated two times).

cardiac tissues) $)^{3,5,11}$. The possibility of the latter scenario has not been investigated in the context of burn injury, especially given that relative to the skeletal and cardiac muscle, the liver is the major organ mobilized to respond to lipids emanating from the adipose tissue after the injury.

Despite this association between the lipolysis and hepatic steatosis post-burn injury, no studies have evaluated the altered lipid molecules and aberrant lipid metabolites that infiltrate the liver to induced hepatic dysfunction. Indeed, hepatic steatosis-associated dysfunction in the liver remains a leading cause of mortality in burn patients ${ }^{9}$. Significant evidence from epidemiological and observational studies have found that burn patients with pre-existing liver disease not only have increased mortality risk from 6 to $27 \%$ of the total population, but post-mortem liver analyses have determined that patients who succumb to the injury demonstrate significant hepatic steatosis and hepatomegaly ${ }^{12}$.

In this study, we investigated the impact of burninduced WAT browning and its associated lipolysis on hepatic steatosis. Our findings show that burn-induced browning leads to the development of hepatic steatosis post-burn injury. Inhibition of the upstream regulator (IL6) and the downstream regulator (UCP-1) of burninduced browning protected mice from the development of hepatic steatosis. Lipidomic analysis in samples taken from both burn patients and post-burn mice revealed the expression of stress-inducing lipids palmitic and stearic acids, which resulted in both hepatic ER stress and impaired fat oxidation in hepatocytes. Thus, our findings establish an important link between WAT browning and hepatic steatosis, which provides a novel target to ameliorate hepatic steatosis and its associated cellular changes after a burn injury.

\section{Results}

WAT browning is associated with lipid accumulation in the livers of post-burn mice

In order to investigate the relationship between burninduced WAT browning and hepatic steatosis, we utilized a mouse model of burn injury. Mice were subjected to a
$30 \%$ total body surface area (TBSA) thermal injury, which successfully induced a hypermetabolic response characterized by body weight loss and accelerated wasting of inguinal WAT (iWAT) and epididymal WAT (eWAT) (Fig. 1a, b). Additionally, the rate of oxygen consumption and lipolysis were significantly elevated in post-burn mice (Fig. 1c, d). This hypermetabolic response to burn injury was accompanied by the stimulation of the browning process, as previously reported in burn patients, namely a phenotypic switch from white to beige fat in subcutaneous iWAT (Fig. 1e), elevated gene and protein expression of the key browning marker UCP1 (Fig. 1f, g) and an increased presence of multilocular cells and UCP1positive histological staining (Fig. 1h).

Interestingly, the aforementioned changes in the adipose tissue were also associated with alterations in the liver of post-burn mice. In fact, we observed a marked increase in liver weight relative to body weight of burned mice compared to controls (Fig. 1i). Histologically, livers from burned mice exhibited substantially greater lipid accumulation compared to those of control mice (Fig. 1j). Mirroring the histological findings, burned mice exhibited almost a 2-fold increase in hepatic TG content compared to control mice (Fig. 1k). To further link the fatty acid accumulation in the liver of post-burn mice to alterations in the adipose tissue and not hepatic de novo lipogenesis (DNL), we measured the expression of key genes associated with DNL. Hepatic expression of key DNL genes (Scd1, Fas, Dgat2, Dak, Ces) were not significantly upregulated in response to burn injury compared to control mice (Fig. 11). Corroborating our gene expression data, we found no significant upregulation of key DNL proteins FAS and SCD1 (Fig. 1m). Together, these findings suggest that the hepatic steatosis observed post-burn injury is a result of the changes in the adipose tissue rather than an increase in hepatic DNL.

\section{Essential roles of IL-6 and UCP1 in mediating burn-induced browning and hepatic steatosis}

Recently, we have uncovered the cytokine interleukin 6 (IL-6) and type 2 macrophages in mediating catecholamine-induced UCP-1 expression and WAT 


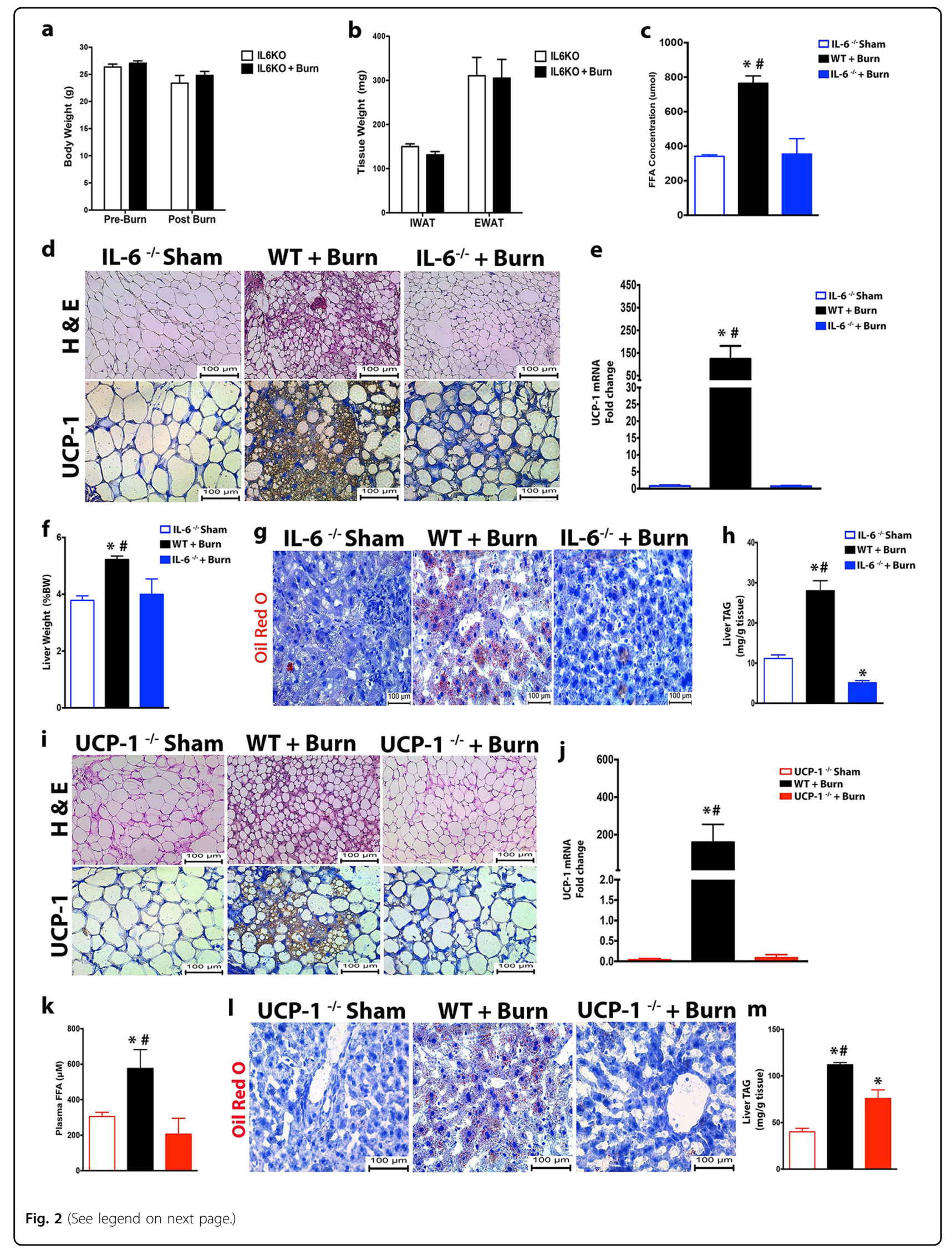


(see figure on previous page)

Fig. $2 \mathrm{IL}-6^{-1-}$ and UCP-1 ${ }^{-1-}$ KO mice are protected from burn-induced browning and hepatic steatosis post-injury. $\mathbf{a}, \mathbf{b}$ Changes in total body (a) and fat (b) weight in IL- $6^{-/-}$burned mice and IL-6 ${ }^{-1-}$ controls. c Plasma concentration of free fatty acids in IL-6 $6^{-1-}$ burned mice and controls. d UCP1 staining in inguinal WAT of WT and IL-6 $6^{-1-}$ burned mice and controls. e Quantitative RT-PCR analysis of browning gene UCP1 in inguinal WAT of wild type (WT) and IL- $6^{-/-}$burned mice and controls. $\mathbf{f}$ Liver weights normalized to body weight of WT and IL-6 $6^{-1-}$ burned mice and controls. $\mathbf{g}$ Oil Red O staining for fat droplets in liver sections from WT and IL- $6^{-/-}$burned mice and controls. $\mathbf{h}$ Triglyceride (TG) content of livers from WT and IL- $6^{-/-}$burned mice and controls. i H\&E and UCP1 staining in inguinal WAT of WT and UCP-1 $1^{-/-}$burned mice and controls. $\mathbf{j}$ Quantitative RT-PCR analysis of browning gene UCP1 in inguinal WAT of WT and UCP- ${ }^{-/-}$burned mice and controls. $\mathbf{k}$ Plasma concentration of free fatty acids in UCP-1 $1^{-1-}$ burned mice and controls. I Oil Red O staining for fat droplets in liver sections from WT and UCP-1 ${ }^{-1-}$ burned mice and controls. $\mathbf{m}$ Triglyceride (TG) content of livers from WT and UCP-1 ${ }^{-1-}$ burned mice and controls. Data represented as mean \pm SEM, $p<0.05$ *significant difference WT burn vs. controls, $p<0.05$ \# WT burn vs. IL- $6^{-/-} /$UCP-1 ${ }^{-1-}(n=7$, biological replicates, experiments repeated two times).

browning during a burn injury ${ }^{3,6,13}$. To directy link WAT browning to the development of hepatic steatosis after a burn injury, we sought to block the two main regulators, IL-6 and UCP-1, involved in post-burn WAT browning. We first used IL-6 whole body $\mathrm{KO}$ (IL- $6^{-1-}$ ) mice, in which mice lack the complete production of systemic IL6 , an upstream regulator previously implicated in both burn and cancer-induced WAT browning (Supplementary Fig. 1a-c). As expected, burn-induced weight loss and adipose tissue wasting was significantly attenuated in $\mathrm{IL}-6^{-1-}$ mice subjected to a burn injury (Fig. 2a, b). IL- $6^{-1-}$ mice were also protected against burn-induced browning as genomic and histological analysis revealed diminished multilocular, UCP1+ adipocytes in the adipose post burn injury (Fig. 2c, d). Additionally, these IL- $6^{-I-}$ mice did not show a significant increase in lipolysis compared to post-burn WT mice (Fig. 2e). In accordance with the above observations made in IL- $6^{-1-}$, inhibition of WAT browning significantly decreased hepatic fat accumulation in these mice post-burn injury. Interestingly, liver weights of IL- $6^{-/-}$were lower compared to WT mice post-burn injury, indicating reductions in lipid infiltration (Fig. 2f). In agreement with our liver weight findings, hepatic lipid infiltration and liver TG content were all reduced in IL- $6^{-1-}$ mice, compared to WT controls post-burn inury (Fig. $2 \mathrm{~g}, \mathrm{~h}$ ).

Furthermore, the browning gene UCP-1 has also been implicated as the downstream regulator of both cold and burn-induced WAT browning ${ }^{14}$. To further implicate WAT browning in post-burn hepatic steatosis, we next utilized UCP-1 KO (UCP-1 ${ }^{-/-}$) mice in which the downstream regulator of post-burn WAT browning, namely, UCP-1 is not expresed. Histological and genomic analysis confirmed that UCP1 expression in WAT was completely ablated in UCP-1 $1^{-/-}$mice (Fig. $2 \mathrm{i}, \mathrm{j}$ ). Notably, loss of the UCP-1 gene in mice also attenuated burninduced lipolysis, reduced hepatic lipid infiltration, and liver TG content (Fig. $2 \mathrm{k}-\mathrm{m}$ ). Together, these findings provide compelling evidence that inhibition of burninduced browning via the deletion of either the upstream or downstream regulators, IL-6 and UCP-1, respectively, attenuates burn-induced hepatic steatosis.

\section{IL-6 receptor blockade and propranolol attenuates WAT browning and hepatic steatosis}

Thus far having shown that removing genes (IL-6, UCP-1) involved in post-burn WAT browning protected mice from hepatic steatosis, we wanted to determine if we could attenuate this adverse post-burn response therapeutically in wild type mice. To accomplish this, we tested the therapeutic potential of Tocilizumab, an IL-6 receptor (IL-6R) blocker that inhibits IL-6 signaling implicated in both burn and cancer-induced WAT browning ${ }^{3,6,13}$. Remarkably, administering the IL-6R blocker in post-burned mice significantly diminished the development of burn-induced hypermetabolism (weight loss, fat wasting) and recovered mitochondrial coupling (Fig. 3a-c). Consistent with reduced hypermetabolism, mice treated with the IL-6R blocker showed reduced UCP1+ cells and decreased gene expression of key browning markers (Fig. 3d, e). The therapeutic effects of IL-6R blocker treatment in the adipose tissue of post-burn mice also carried over to the liver, where we observed reductions in markers of hepatic steatosis (Fig. $3 \mathrm{f}-\mathrm{h}$ ). Similarly, we also examined the potential therapeutic effect of inhibiting WAT browning in post-burn mice via the catecholamine antagonist propranolol, an FDA approved drug currently in use in trauma and burn patients for cardiac purposes ${ }^{15,16}$. Interestingly, treatment of propranolol in post-burned mice significantly attenuated burn-induced hypermetabolism, WAT browning, and hepatic steatosis (Supplementary Fig. 2a-h). Taken together these findings provide further compelling evidence that WAT browning mediates post-burn hepatic steatosis and that blocking this process could serve as a potential therapeutic strategy to counteract its adverse effects on the liver.

\section{Elevated expression of ER stress inducing lipids suppress hepatic fat oxidation after a burn injury}

Having established hepatic steatosis occurs following burn-induced browning and lipolysis, we next focused on identifying and assessing the impact of the released lipids from the adipose tissue on hepatic function and fat oxidation. Lipid profiling in samples taken from both burn 


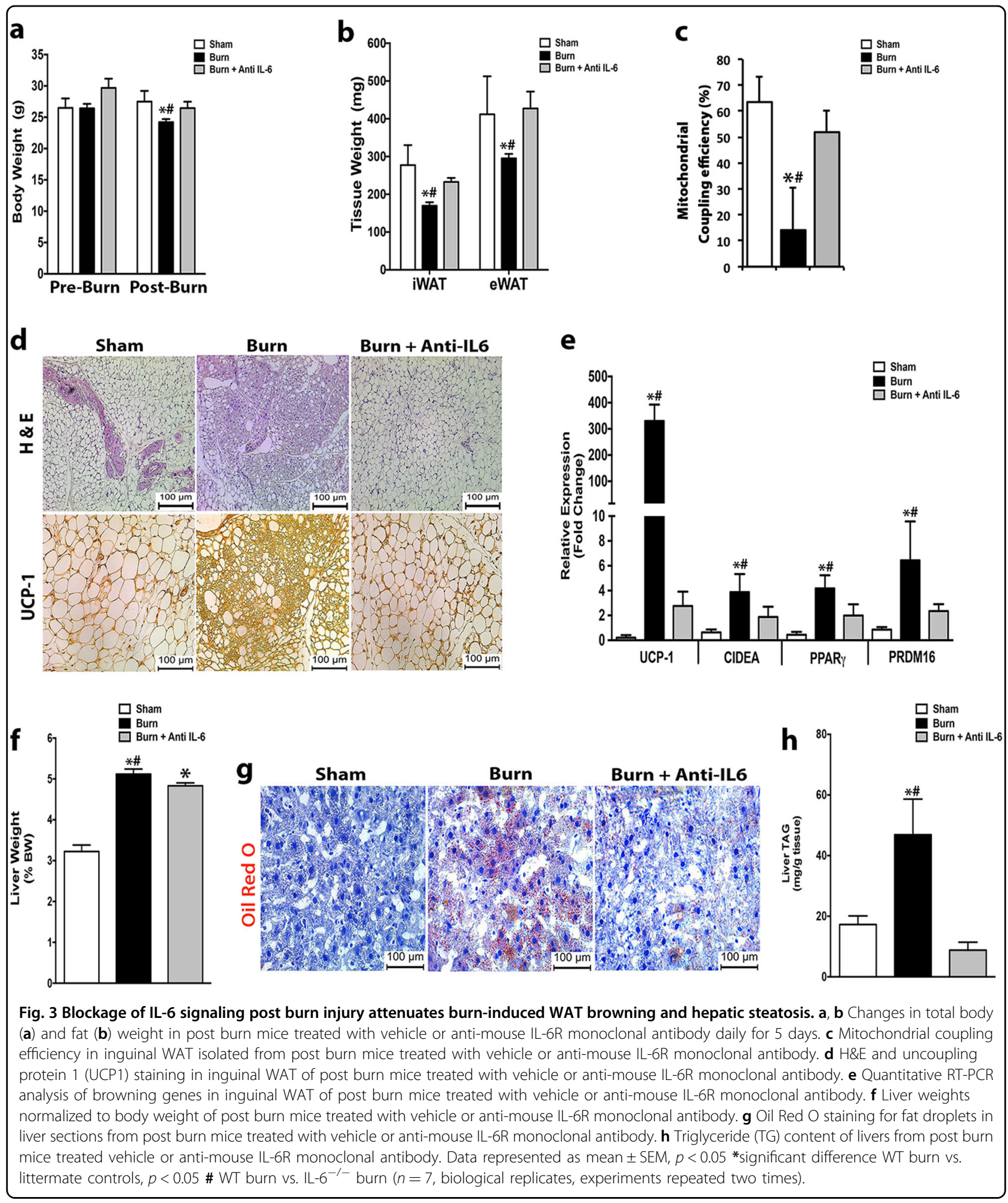

patients and post-burn mice using chromatography-mass spectrometry (LC-MS)-based global lipidomic analysis, revealed changes to a total of 9 (burn patients) and 11 (mice) circulating fatty acids in response to the injury
(Fig. 4a, b). It has been reported that the accumulation of specific lipids (palmitic and stearic acid) is damaging in regards to hepatocyte function and survival, as these lipids lead to the activation of the unfolded protein response 


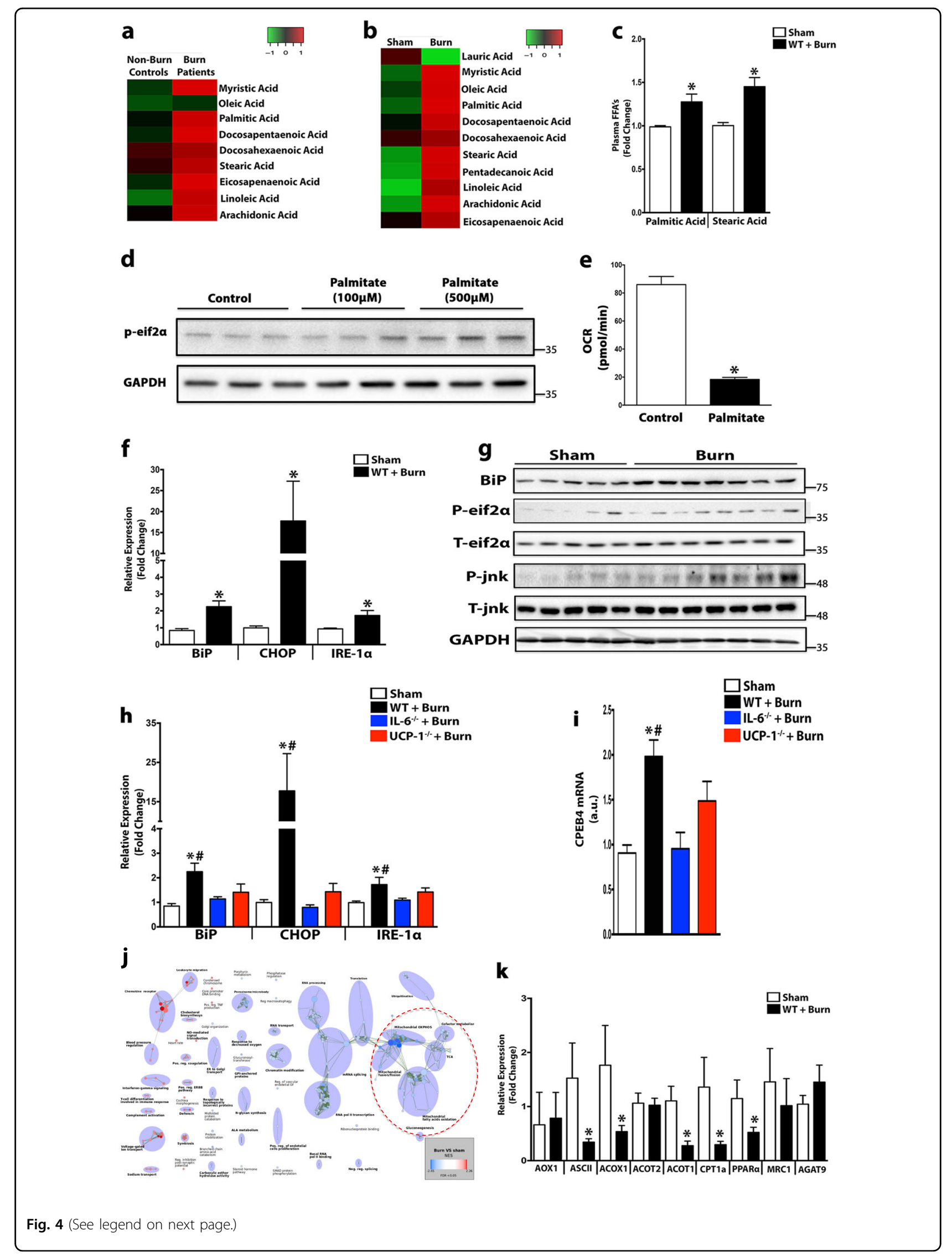


(see figure on previous page)

Fig. 4 Lipodomic profiling after a burn injury reveals upregulation of ER stress inducing lipids. $\mathbf{a}$, $\mathbf{b}$ Heat map display of lipid species within the plasma taken from burn patients and post-burn mice. c Quantification of FFAs (Palimitic and Stearic) in the plasma of wild type burned mice and controls. $\mathbf{d}$ Immunoblot of ER stress marker p-eif2a in HepG2 cells exposed to vehicle or Palimitate $(100 \mu \mathrm{m}$ or $500 \mathrm{~m})$ for $24 \mathrm{~h}$. e Oxygen Consumption Rate (OCR) in HepG2 cells exposed to vehicle or Palimitate $(500 \mu \mathrm{m})$ for $24 \mathrm{~h}$. $\mathbf{f}$ Quantitative RT-PCR analysis of ER stress/UPR gene expression from livers of wild type (WT) burned mice and controls. $\mathbf{g}$ Immunoblot analysis of ER stress/UPR proteins in liver samples of wild type (WT) burned mice and controls. $\mathbf{h}$, i Quantitative RT-PCR analysis ER stress/UPR and CPEB4 gene expression from the livers of UCP-1 $1^{-/-}$and IL-- ${ }^{-/-}$burned mice and controls. $\mathbf{j}$ Parametric analysis of gene-set enrichment (PAGE) of the most highly upregulated (red) and down-regulated (blue) mitochondrial genes in livers of wild type (WT) burned mice and controls. $\mathbf{k}$ Quantitative RT-PCR analysis of beta-oxidation genes in the livers of wild type (WT) burned mice and controls. Data represented as mean \pm SEM, $p<0.05 *$ significant difference WT burn vs. controls $(n=8$, biological replicates, experiments repeated two times).

$(\mathrm{UPR})^{17}$. We found a $\sim 1.5$ fold increase in the plasma expression of palmitic and stearic acid after a burn injury (Fig. 4c), which led us to postulate that ER stress may be at play in the context of browning-induced hepatic dysfunction. Indeed, treatment of in vitro hepatocytes with palimitate resulted in ER stress activation and decreased mitochondrial respiration (Fig. 4d-e). Similarly, in livers from mice that have undergone WAT browning and developed hepatic steatosis after a burn injury, we saw increased gene and protein expression of ER stress markers BiP, CHOP, IRE-1 $\alpha$, and phosphorylated forms of JNK and eIF2 $\alpha$ (Fig. 4f, g). Conversely, both $\mathrm{UCP} 1^{-1-}$ and IL- $6^{-1-}$ mice that were protected from both burninduced WAT browning and hepatic steatosis, also showed reductions in hepatic ER stress (Fig. 4h). Additionally, the cytoplasmic polyadenylation element-binding (CPEB-4) protein, which is only synthesized following ER stress, was upregulated in post-burn mice and attenuated in post-burn mice where WAT browning was inhibited (Fig. 4i).

Furthermore, studies have also reported that hepatic ER stress can exacerbate organ steatosis by inhibiting genes involved in lipid catabolism ${ }^{18,19}$. To directly assess this, we performed microarray analysis in livers from burned mice, which showed a substantial reduction in genes involved in oxidative metabolism and lipid transport (Fig. 4h). Confirming our microarray data, expression of genes associated with $\beta$-oxidation such as proliferator-activated receptor alpha (Ppar $\alpha)$, carnitine palmitoyltransferase $1 \alpha$ $(\mathrm{Cpt} 1 \alpha)$, and peroxisomal and microsomal oxidation genes (Acox1, Aox1) were also suppressed in the livers of post-burn mice (Fig. 4i). These findings demonstrate that burn-induced WAT browning and the associated release of lipids not only augments the development of hepatic steatosis, but also impairs the ability of the liver to mitigate the infiltration of these lipids by suppressing fatty acid oxidation.

\section{Hepatic ER stress facilitates increased ER-mitochondrial coupling after a burn injury}

Having identified reduced hepatic fat oxidation after a burn injury, we next sought to uncover how hepatic ER stress mediated this response at the organelle level. Recently, it has been reported that hepatic lipid transport and metabolism have been shown to be enriched in contact membranes between the ER and mitochondria termed the mitochondria-associated membrane (MAM), and that hepatic ER stress facilitates increased MAM formation ${ }^{20-22}$.

Indeed, we not only found the deregulation of genes involved in maintaining normal MAM formation ${ }^{22,23}$ (Supplementary Fig. 3a, b), but we also observed a higher degree of ER tethering to the mitochondria in mice subjected to a burn (Fig. 5a, b). To further confirm increased hepatic MAM enrichment and ER-mitochondria coupling, we employed an organelle targeted in situ proximity ligation assay (PLA). Using in situ PLA by targeting the ER organelle surface marker inositol 1,4,5-trisphosphate receptor $\left(\mathrm{IP}_{3} \mathrm{R}\right)$ and mitochondrial surface marker voltage dependent anion channel (VDAC), we were able to quantify the level of interaction between the two organelles. Confirming our TEM observations, we found significantly increased ER-mitochondria cross-talk in liver sections from burned mice (Fig. 5c, d). Additionally, we found an increased expression of the MAM enriched protein $\mathrm{IP}_{3} \mathrm{R} 1$ in liver lysates of burned mice (Fig. 5e).

To confirm the changes in hepatic ER-Mitochondrial coupling resulted from hepatic ER stress as has previously been reported ${ }^{22}$, we examined the effect of acute ER stress in the liver in vivo by administration of the ER stressinducing drug tunicamycin as a "second hit" in post-burn mice. Tunicamycin treatment in post-burn mice resulted in a higher degree of ER-mitochondria interaction and increased expression of the MAM enriched protein IP3R1 than what we observed earlier in burned mice alone (Fig. 5f, g). The greater degree of ER-mitochondrial interaction observed in burn mice treated with tunicamycin was corroborated by in situ proximity ligation assay (Fig. 5h, i). In parallel, we also performed genome-wide microarray analysis in the livers of burn mice treat with tunicamycin, which showed greater suppression of genes involved in $\beta$-oxidation compared to burned mice alone (Fig. 5j). These ER-stress induced alterations at the organelle level also resulted in hepatic dysfunction as 

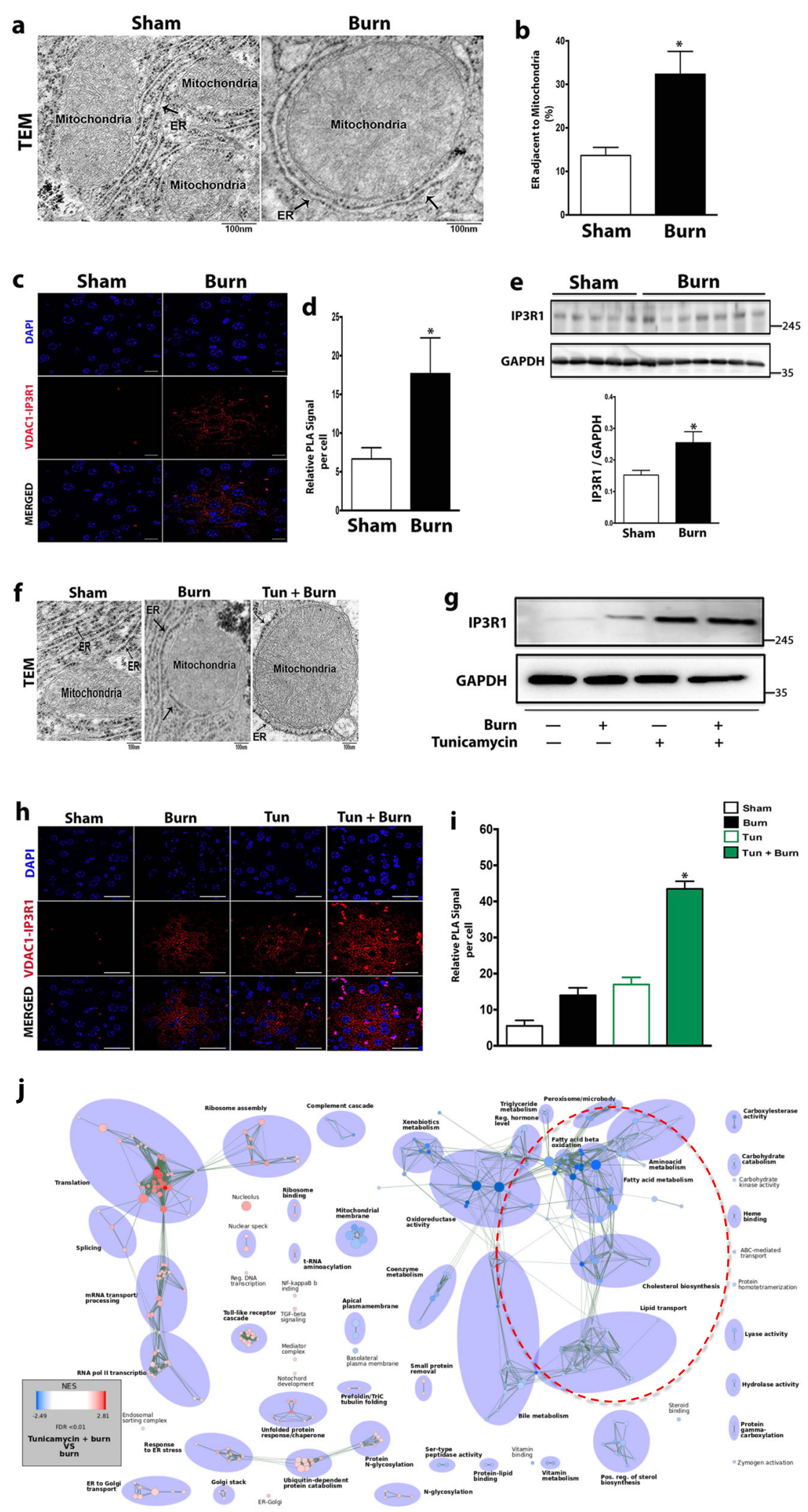

Fig. 5 (See legend on next page.) 
(see figure on previous page)

Fig. 5 Hepatic ER stress post-burn injury leads to increased ER-Mitochondrial interaction. $\mathbf{a}$, b Representative TEM images and quantification ( $s$ cale bare $=100 \mathrm{~nm}$ ) illustrating ER-Mitochondria tethering in liver sections derived from wild type (WT) burned mice and controls.

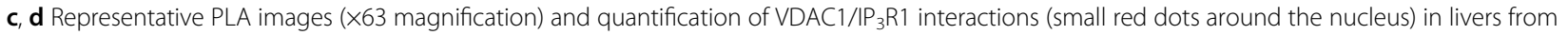
wild type (WT) burned mice and controls. e Representative western blot and quantification of MAM enriched protein IP ${ }_{3} \mathrm{R} 1$ in livers of wild type (WT) burned mice and controls. $\mathbf{f}$ Representative TEM images (scale bare $=100 \mathrm{~nm}$ ) illustrating ER-mitochondria tethering of liver sections derived Sham, Burn, and Tun + Burn mice. $\mathbf{g}$ Representative western blot of MAM enriched protein IP3R1 in livers of Sham, Burn, Tun., and Tun + Burn mice. $\mathbf{h}, \mathbf{i}$ Representative PLA images ( $\times 63$ magnification) and quantification of VDAC1/IP $R 1$ interactions (small red dots around the nucleus) in livers of Sham, Burn, Tun., and Tun + Burn mice. $\mathbf{j}$ Parametric analysis of gene-set enrichment (PAGE) of the most highly upregulated (red) and downregulated (blue) mitochondrial genes in livers of burned mice and Tun + Burn mice. Data represented as mean \pm SEM, $p<0.05 *$ significant difference WT burn vs. control, $p<0.05$ \# WT burn vs. Tun + Burn ( $n=8$, biological replicates, experiments repeated two times).

markers of hepatic damage such as alanine aminotransferase (ALT), hepatocyte proliferation (as determined by Ki-67 staining), and apoptosis, as determined by TUNEL staining and caspase 3 activation, were significantly upregulated in both post-burn mice and burn mice treated with tunicamycin (Supplementary Fig. 4a-d). Collectively, our findings indicate that hepatic ER stress facilitates alterations at the organelle level between the ER and mitochondria in order to suppress fat oxidation.

\section{Cell-permeant $I P_{3} R$ peptide potently blocks ER stress induced hepatocyte apoptosis}

Hepatic ER stress and increased MAM enrichment have also been implicated in mediating hepatocyte apoptosis. Indeed, others and we have previously reported that increased hepatic ER stress and $\mathrm{IP}_{3} \mathrm{R} 1$ facilitates increased calcium shuttling from the ER to the mitochondria to induce cellular apoptosis ${ }^{22}$. During cellular apoptosis, it has also been reported that Cytochrome $\mathrm{C}$ exits the mitochondria and binds directly and selectively to $\mathrm{IP}_{3} \mathrm{R} 1$ in the closely adjacent ER, thereby amplifying calciumdependent apoptosis ${ }^{24,25}$. Using the drug thapsigargin, which facilitates ER-calcium depletion and ER stress, we aimed to study the therapeutic effect of a cell permeable peptide $\left(\mathrm{IP}_{3} \mathrm{R}-\mathrm{CYT}\right)$ that displaces Cytochrome $\mathrm{C}$ from $\mathrm{IP}_{3} \mathrm{R}$, thereby blocking calcium-induced apoptosis and $\mathrm{ER}$ stress (Fig. 6a-c). We were able to abrogate key ER stress markers, specifically phosphorylated eIF $2 \alpha$ and CHOP, induced by thapsigargin with the use of the $\mathrm{IP}_{3} \mathrm{R}$ peptide that displaces Cytochrome $\mathrm{C}$ from $\mathrm{IP}_{3} \mathrm{R}$ (Fig. 6b, c, e). Treating these HEPG2 cells with the $\mathrm{IP}_{3} \mathrm{R}$ peptide also attenuated ER stress induced mitochondrial damage (Fig. 6d, f). Thus, our data demonstrate that directly targeting proteins that mediate ER-mitochondria cross talk might be a promising therapeutic strategy to mitigate the adverse cellular effects of hepatic ER stress after a burn injury.

\section{Discussion}

Current understanding of the activation of WAT browning in hypermetabolic conditions (burns, cancer) is poorly understood, and whether such browning has deleterious effects on outcome and organ function are even less known. In this study, we provide evidence to support the adverse effects of beige fat in a burn injury model of hypermetabolism. We provide evidence that browning of WAT after a burn injury triggers lipolysis, which in turn leads to an increased blood lipid profile that culminates in hepatic steatosis and dysfunction via ER stress (Fig. 7). This effect appeared to be browning mediated as blockage of WAT browning via the upstream regulator (IL-6) and the downstream regulator (UCP-1) in mice attenuated hepatic steatosis after a burn injury. We also identified the pathogenesis of the infiltrated lipids for the liver, as lipidomic profiling of the plasma of both burned mice and patients revealed the expression of palmitic and stearic acid, which mediated hepatic ER stress and the suppression of fat oxidation. Mechanistically, we also uncovered abnormal ER-mitochondria cross talk via MAM enrichment and $\mathrm{IP}_{3} \mathrm{R} 1$ expression as the cellular mechanisms behind lipid-induced hepatic dysfunction and apoptosis.

To date, WAT browning has been described as a beneficial event in the context of obesity and diabetes, as it has been shown to promote weight loss and improve insulin sensitivity in both metabolic conditions ${ }^{26,27}$. However, browning in the context of cancer has recently been shown to be detrimental, as it facilitates cancerinduced wasting and cachexia ${ }^{3,5}$. Burn injury, which induces a similar hypermetabolic state as seen in cancer, was also recently shown to induce browning, although whether such browning is actually detrimental in the burn context is still unknown. Here, we show that burn injury induced browning mediates hepatic steatosis and adverse alterations at the cellular level. The liver is a focal point for lipid mobilization that oxidizes lipids for glucose during fasting and packages these lipids into lipoproteins for peripheral tissues ${ }^{28,29}$. In the lipid-accumulated livers of post burn mice, we observed the activation of ER stress, which was non-existent in burned mice where browning was inhibited. It has been previously reported in studies outside of burns that ER stress leads to lipid accumulation 


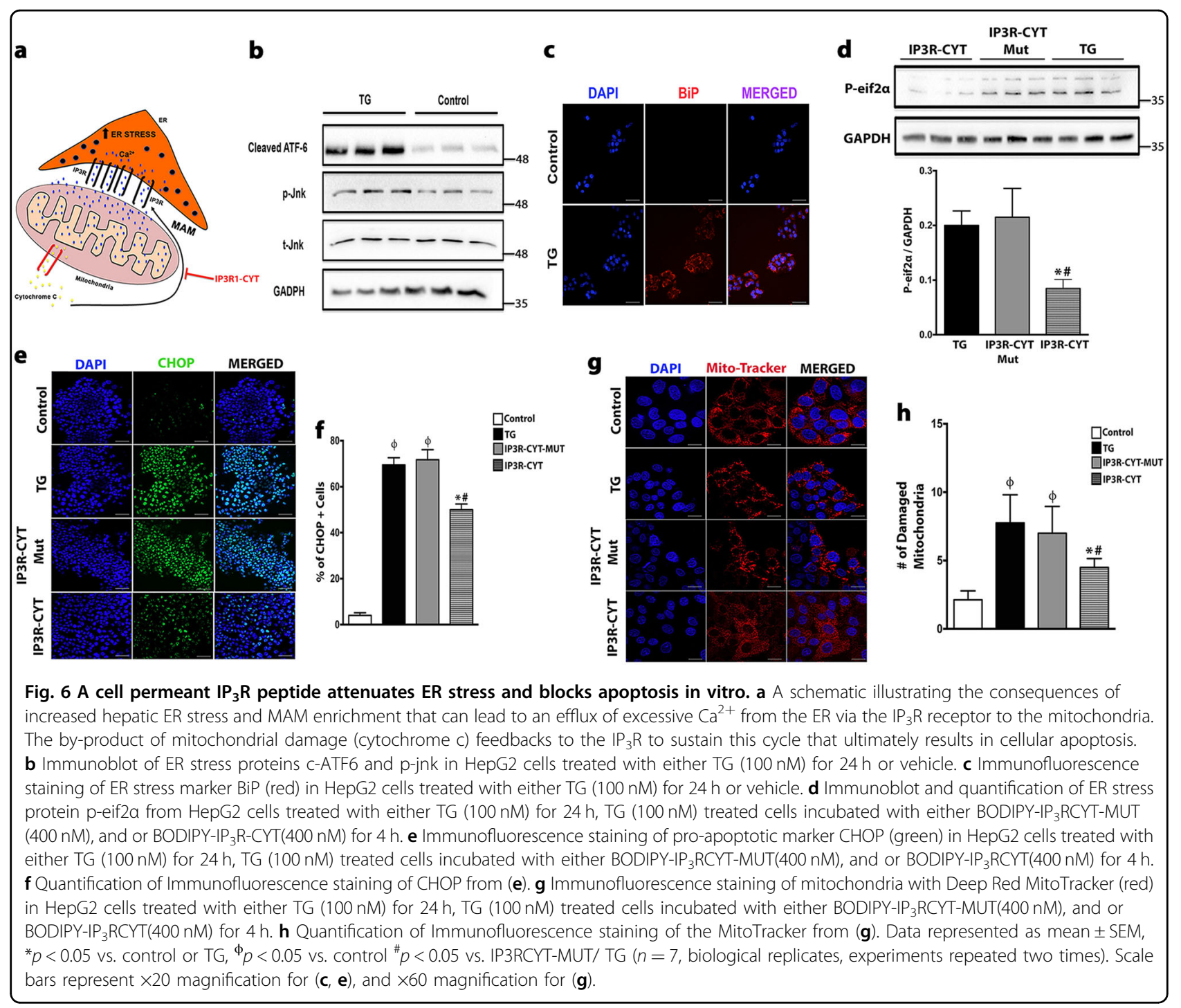

and hepatic steatosis via the inhibition of betaoxidation $^{18,30}$. Indeed, our findings in the livers of postburn mice corroborate these past findings and indicate that lipid-induced hepatic ER stress impairs fat oxidation, which in turn further facilitates fat accumulation in this organ.

Our study also aimed to develop a deeper understanding of the cellular changes that take place after a burn injury in the hopes of resolving how browninginduced hepatic steatosis and ER stress leads to suppression of hepatic fat oxidation. Although the precise mechanisms of how ER stress fosters the suppression of fat oxidation remains elusive, it likely involves the physical interaction between the ER and mitochondria, namely the MAM. The MAM not only establishes a physical and functional connection between the ER and the mitochondria, but also regulates fat metabolism and calcium signaling $^{31}$. The attendant crosstalk between the ER and mitochondria appears to be vital for cell integrity, fat metabolism, death, and maintenance of function ${ }^{32}$. In fact, it has recently been shown that abnormal communication between the ER and mitochondria in obesity has damaging effects on mitochondrial and cellular function ${ }^{33}$. Our findings in which after a burn injury hepatic ER stress results in greater ER-mitochondrial interaction and the expression of the MAM enriched $\mathrm{IP}_{3} \mathrm{R}$ protein support these findings albeit in the context of a response to a traumatic injury. It has also been reported that calcium depletion from the $E R$ via the $I_{3} R$ receptor leads to calcium overload in the mitochondria, which in turn activates a feed-forward cascade whereby released cytochrome $\mathrm{c}$ interacts with the $\mathrm{IP}_{3} \mathrm{R}$ receptor to further amplify calcium release and eventually lead to hepatocyte apoptosis $^{24,25}$. The hepatocyte apoptosis after a burn injury we observed likely resulted from ER stress induced $\mathrm{IP}_{3} \mathrm{R} 1$ over expression, as our in vitro findings showed that hepatocyte ER stress and mitochondrial dysfunction 


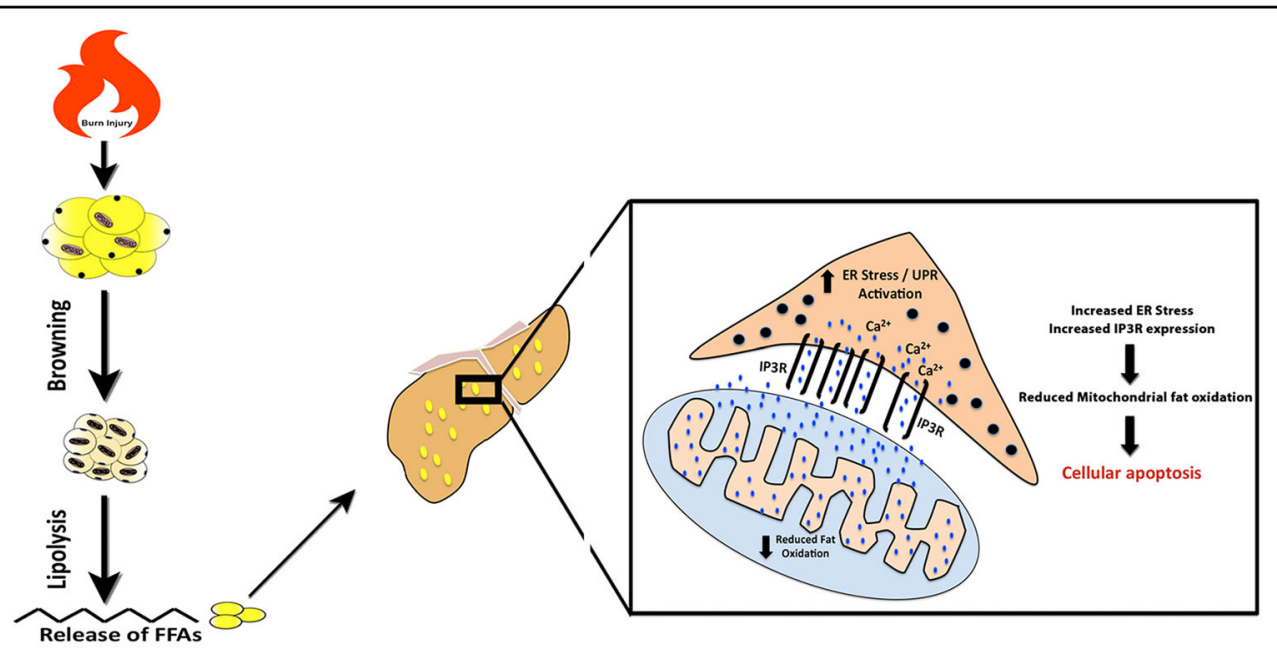

Fig. 7 Schematic diagram illustrating the consequences of WAT browning and its associated lipotoxicity for the liver after a burn injury. Following a burn injury you have the activation of the browning process whereby the white adipose tissue converts to beige fat. Pro-inflammatory FFAs released from beige fat than travel to the liver causing hepatic steatosis and hepatic dysfunction by activating the ER stress response in hepatocytes. Hepatic ER stress then leads to increased expression of the MAM enriched protein $\mathrm{P}_{3} \mathrm{R} 1$, which leads to a greater ER-Mitochondria interaction and facilitates $\mathrm{Ca}^{2+}$ transfer from $\mathrm{ER}$ (via $\mathrm{IP}_{3} \mathrm{R} 1$ ) to the mitochondria. This, in turn, can lead to reductions in mitochondrial fat oxidation. If this chronic ER stress mediated by lipid infiltration is not mitigated it can ultimately result in cellular apoptosis.

were both abrogated by a cell permeant peptide that displaces cytochrome $\mathrm{c}$ from $\mathrm{IP}_{3} \mathrm{R}$. Therefore, we suggest that targeting proteins that regulate the ER-mitochondrial interface in order to restore normal function, maybe a useful approach to mitigate the effects of lipid-induced hepatocyte ER stress and cell death.

Our findings also highlighted the potential therapeutic benefits of blocking the browning process after a burn injury for the liver. Based on our previous findings in which we uncovered IL- 6 and type 2 macrophages as the regulators of post-burn browning, we envisioned that blocking IL-6 signaling via the use of an IL-6 receptorneutralizing (IL-6R) antibody might be therapeutically beneficial in alleviating both WAT browning and hepatic steatosis. Indeed, blocking the IL-6R was able to inhibit adipose browning and its associated hepatic steatosis in post-burned mice. These findings are critical because most of the work in blocking WAT browning has focused on blocking the $\beta$-adrenergic receptors, leaving other potential avenues relatively unexplored. Interestingly, it has been reported that patients receiving the IL-6R neutralizing mAb Actemra (tocilizumab) for rheumatoid arthritis show side-effects like an increase in body weight and marked hypercholesterolemia during the treatment period, which maybe a result of an inhibition of WAT browning in these patients who also have chronic IL-6 production. Thus, while our study broadens the understanding of WAT browning in contexts where it plays a detrimental rather than a beneficial role, further studies are warranted to examine the effects of browning in other tissues outside the liver.

\section{Materials and methods \\ Human samples}

Patients admitted to the Ross Tilley Burn Centre at Sunnybrook Hospital (Toronto, Canada) or non-burn patients undergoing elective surgery were consented preoperatively for blood collection. Approval for our study was obtained from the Research Ethics Board at Sunnybrook Hospital.

\section{Animals}

Male C57BL/6 and IL6 ${ }^{-1-}$ mice (Jackson, USA) were housed at thermoneutral temperature $\left(28^{\circ} \mathrm{C}\right)$ and cared in accordance with the Guide for the Care and Use of Laboratory Animals. Congenic lines of $\mathrm{UCP}-1^{-1-}$ mice were generated by backcross matings of heterozygous $(+/-)$ mice on a mixed 129/SvPas and C57BL/6J background with 129/SvImJ and C57BL/6J mice as previously described (Jackson, USA). In this study, all wild-type mice are C57BL/6J and are referred to as wild type (WT) and all UCP-1 mutant mice are on the C57BL/6J background and are referred to as $\mathrm{UCP1}^{-1-}$. Randomization of animals was done by the animal tech that divided the animal cohorts into groups of 5 in a cage after arrival to the facility. These cohorts were then treated as described in the paper. Mice were housed and cared in accordance with the Guide for the Care and Use of Laboratory Animals and procedures approved by the Sunnybrook Research Institute Animal Care Committee under AUP 467 (Toronto, Ontario, Canada). Due to the nature of the burn injury, size estimates and the number animals per group were done in consultation with the Animal Care 
Committee at Sunnybrook Research Institute. All experiments were performed with 2 month old male WT and $\mathrm{UCP} 1^{-1-}$ mice. Mice receiving an injury were subjected to a $30 \%$ total body surface area (TBSA) full thickness burn. All mice were subsequently housed individually in sterile cages and fed ad libitum until sacrifice. Tissues were harvested 5 days post burn injury and used for analysis.

\section{Mouse burn injury model}

Mice were anaesthetized using ketamine $(0.15 \mathrm{mg} / \mathrm{g})$ and xylazine $(0.01 \mathrm{mg} / \mathrm{g})$. The dorsum of the trunk was shaved and $1.5 \mathrm{ml}$ Ringer's Lactate was injected subcutaneously along the spine and $0.5 \mathrm{ml}$ Ringer's Lactate was injected intraperitoneally. Mice were placed in a mold that exposed $30 \%$ total body surface area of the shaved dorsum. A full thickness cutaneous scald burn was administered by lowering the mold into a $98^{\circ} \mathrm{C}$ water bath for $10 \mathrm{~s}$. Mice in the sham group were shaved, anaesthetized and received Ringer's Lactate.

\section{Histology and immunohistochemistry}

Adipose tissue was immediately fixed in $10 \%$ formalin and then maintained in $70 \%$ ethanol prior to paraffin embedding. Subsequently, tissues were sectioned and stained with Haematoxylin and Eosin (H\&E) or incubated with UCP1 (Sigma) antibody followed by DAB staining. Livers from mice were isolated and immediately frozen in a tissue freezing medium (O.C.T. compound, Tissue-Tek) and stored at $-80^{\circ} \mathrm{C}$ until staining. Imaging was performed on a LSM confocal microscope (Zeiss, Germany). The investigator was blinded after sample collection for purposes of histology imaging and analysis.

\section{Quantitative PCR}

Total RNA isolated from adipose tissue, and primary macrophages was analyzed by quantitative RT-PCR. RNA was isolated from tissue and cells using TRIzolchloroform (Life Technologies) with subsequent purification using the RNeasy Kit (Qiagen) according to the manufacturer's instructions. RNA (2 mg) was transcribed to cDNA using the high capacity cDNA reverse transcription kit (Applied Biosystems). Real-time quantitative PCR was performed using the Applied Biosystems Step One Plus Real-Time PCR System. Primer sequences used are available upon request.

\section{Microarray analysis}

The liver was dissected from each mouse and homogenized, total RNA was extracted using a QIAGEN kit according to the manufacturer's instructions. For gene profile analysis, RNA quality was assessed with a Bioanalyzer (Agilent Technologies), and samples with an RNA purity greater than 1.8 were included for array. cDNA was generated and hybridized onto the Affymetrix Mouse Gene 1.0 ST chips. Analysis of gene expression was performed using Parktec Genotyping Suite and Ingenuity Systems Software.

\section{Statistical analysis}

All data are presented as mean \pm s.e.m and analyzed using Prism (Graphpad). Statistical significance was determined using a Student's t-test or one-way ANOVA followed by a Bonferroni posthoc tests were used where appropriate. A $p$-value of $<0.05$ was considered to be statistically significant, and is presented as $*(p<0.05)$. Samples were excluded as outliers if they were 2 standard deviations above or below the mean. Tests were done that showed the variance was equal among the different groups.

Other Detailed experimental procedures and protocols are described in the Supplementary Material.

\section{Acknowledgements \\ The authors would like to thank Steven Doyle for sample preparation and assistance with electron microscopy, Petia Stefanova for histological analysis, Carly Knuth for editing assistance. This study was supported by National Institutes of Health R01-GM087285-01, DK104867 (SB). Canadian Institutes of Health Research (CIHR) grant \#123336. A. A. is a recipient of the Vanier Canada Graduate Scholarship funded by the Canadian Institutes of Health Research.}

\section{Author details}

${ }^{1}$ Faculty of Medicine, University of Toronto, Toronto, ON, Canada. ${ }^{2}$ Biological Sciences, Sunnybrook Research Institute, Toronto, ON, Canada. ${ }^{3}$ Department of Biochemistry and Molecular Biology, University of Texas Health Science Center at Houston, Houston, TX, USA. ${ }^{4}$ Department of Psychiatry and Behavioral Sciences, Johns Hopkins University School of Medicine, Baltimore, MD, USA. ${ }^{5}$ Ross Tilley Burn Centre, Sunnybrook Hospital, Toronto, ON, Canada. ${ }^{6}$ Department of Surgery, Division of Plastic Surgery and Department of Immunology, University of Toronto, Toronto, ON, Canada

Conflict of interest

The authors declare that they have no conflict of interest.

\section{Publisher's note}

Springer Nature remains neutral with regard to jurisdictional claims in published maps and institutional affiliations.

Supplementary Information accompanies this paper at (https://doi.org/ 10.1038/s41419-019-2103-2).

Received: 23 July 2019 Revised: 19 September 2019 Accepted: 22 October 2019

Published online: 18 November 2019

\section{References}

1. Jeschke, M. G. et al. Long-term persistance of the pathophysiologic response to severe burn injury. PLOS ONE 6, e21245 (2011).

2. Petruzzelli, M. \& Wagner, E. F. Mechanisms of metabolic dysfunction in cancerassociated cachexia. Genes Dev. 30, 489-501 (2016).

3. Petruzzelli, M. et al. A switch from white to brown fat increases energy expenditure in cancer-associated cachexia. Cell Metab. 20, 433-447 (2014).

4. Patsouris, D. et al. Burn induces browning of the subcutaneous white adipose tissue in mice and humans. Cell Rep. 13, 1538-1544 (2015).

5. Kir, S. et al. Tumour-derived PTH-related protein triggers adipose tissue browning and cancer cachexia. Nature 513, 100-104 (2014). 
6. Abdullahi, A. et al. Alternatively activated macrophages drive browning of white adipose tissue in burns. annals of surgery, https://doi.org/10.1097/ SLA.0000000000002465 (2017).

7. Abdullahi, A. \& Jeschke, M. G. White adipose tissue browning: a double-edged sword. Trends Endocrinol. Metab. 27, 542-552 (2016).

8. Jeschke, M. G., Micak, R. P., Finnerty, C. C. \& Herndon, D. N. Changes in liver function and size after a severe thermal injury. Shock 28, 172-177 (2007).

9. Jeschke, M. G. The hepatic response to thermal injury: is the liver important for postburn outcomes? Mol. Med 15, 337-351 (2009).

10. Jeschke, M. G., Barrow, R. E. \& Herndon, D. N. Extended hypermetabolic response of the liver in severely burned pediatric patients. Arch. Surg. 139, 641-647 (2004).

11. Dong, M. et al. Cold exposure promotes atherosclerotic plaque growth and instability via UCP1-dependent lipolysis. Cell Metab. 18, 118-129 (2013).

12. Price, L. A., Thombs, B., Chen, C. L. \& Milner, S. M. Liver disease in burn injury: evidence from a national sample of 31,338 adult patients. J. Burns Wounds 7, e1 (2007).

13. Abdullahi, A. et al. IL-6 Signal from the bone marrow is required for the browning of white adipose tissue post burn injury. Shock 47, 33-39 (2017).

14. Nedergaard, J. et al. UCP1: the only protein able to mediate adaptive nonshivering thermogenesis and metabolic inefficiency. Biochim. Biophys. Acta. 1504, 82-106 (2001).

15. Gauglitz, G. G, Williams, F. N, Herndon, D. N. \& Jeschke, M. G. Burns: where are we standing with propranolol, oxandrolone, recombinant human growth hormone, and the new incretin analogs? Curr. Opin. Clin. Nutr. Metab. Care.14 176-181 2011

16. Rosenberg, L. et al. Does acute propranolol treatment prevent posttraumatic stress disorder, anxiety, and depression in children with burns? J. Child Adolesc. Psychopharmacol. 28, 117-123 (2018).

17. Malhi, H., Bronk, S. F., Werneburg, N. W. \& Gores, G. J. Free fatty acids induce JNK-dependent hepatocyte lipoapoptosis. J. Biol. Chem. 281, 12093-12101 (2006).

18. Rutkowski, D. T. et al. UPR pathways combine to prevent hepatic steatosis caused by ER stress-mediated suppression of transcriptional master regulators. Dev. Cell 15, 829-840 (2008).

19. Yamamoto, K. et al. Induction of liver steatosis and lipid droplet formation in ATF6alpha-knockout mice burdened with pharmacological endoplasmic reticulum stress. Mol. Biol. Cell 21, 2975-2986 (2010).

20. Walter, L. \& Hajnoczky, G. Mitochondria and endoplasmic reticulum: the lethal interorganelle cross-talk. J. Bioenerg. Biomembr. 37, 191-206 (2005).
21. Ardail, D. et al. The mitochondria-associated endoplasmic-reticulum subcompartment (MAM fraction) of rat liver contains highly active sphingolipidspecific glycosyltransferases. Biochem J. 371, 1013-1019 (2003).

22. Rusinol, A. E, Cui, Z, Chen, M. H. \& Vance, J. E. A unique mitochondriaassociated membrane fraction from rat liver has a high capacity for lipid synthesis and contains pre-Golgi secretory proteins including nascent lipoproteins. J. Biol. Chem. 269, 27494-27502 (1994).

23. Shiao, Y. J., Lupo, G. \& Vance, J. E. Evidence that phosphatidylserine is imported into mitochondria via a mitochondria-associated membrane and that the majority of mitochondrial phosphatidylethanolamine is derived from decarboxylation of phosphatidylserine. J. Biol. Chem. 270, 11190-11198 (1995).

24. Boehning, D. et al. Cytochrome $\mathrm{c}$ binds to inositol $(1,4,5)$ trisphosphate receptors, amplifying calcium-dependent apoptosis. Nat. Cell Biol. 5, 1051-1061 (2003).

25. Boehning, D., van Rossum, D. B., Patterson, R. L. \& Snyder, S. H. A peptide inhibitor of cytochrome c/inositol 1,4,5-trisphosphate receptor binding blocks intrinsic and extrinsic cell death pathways. Proc. Natl Acad. Sci. USA 102, 1466-1471 (2005)

26. Bostrom, P. et al. A PGC1-alpha-dependent myokine that drives brown-fat-like development of white fat and thermogenesis. Nature 481, 463-468 (2012).

27. Feldmann, H. M., Golozoubova, V., Cannon, B. \& Nedergaard, J. UCP1 ablation induces obesity and abolishes diet-induced thermogenesis in mice exempt from thermal stress by living at thermoneutrality. Cell Metab. 9, 203-209 (2009).

28. Ponziani, F. R., Pecere, S., Gasbarrini, A. \& Ojetti, V. Physiology and pathophysiology of liver lipid metabolism. Expert Rev. Gastroenterol. Hepatol. 9 1055-1067 (2015).

29. Mayes, P. A. \& Felts, J. M. Regulation of fat metabolism of the liver. Nature $\mathbf{2 1 5}$ 716-718 (1967)

30. DeZwaan-McCabe, D. et al. ER stress inhibits liver fatty acid oxidation while unmitigated stress leads to anorexia-induced lipolysis and both liver and kidney steatosis. Cell Rep. 19, 1794-1806 (2017).

31. Betz, C. et al. Feature Article: mTOR complex 2-Akt signaling at mitochondriaassociated endoplasmic reticulum membranes (MAM) regulates mitochondrial physiology. Proc. Natl Acad. Sci. USA 110, 12526-12534 (2013).

32. Hacki, J. et al. Apoptotic crosstalk between the endoplasmic reticulum and mitochondria controlled by Bcl-2. Oncogene 19, 2286-2295 (2000).

33. Arruda, A. P. et al. Chronic enrichment of hepatic endoplasmic reticulummitochondria contact leads to mitochondrial dysfunction in obesity. Nat. Med 20, 1427-1435 (2014) 